\title{
Physician, heal thyself
}

"M r. President is a rancher, ignorant about the way science is made. For him, to understand that science is a fundamental part of the country's culture is practically impossible." This assertion was made last July by Antonio Peña, past president of the Mexican Academy of Sciences, to criticize his government's limited support for science. Peña's statement is typical of what many scientists in countries wealthy enough to spend on research but not fully developednations sometimes referred to as 'emerging economies'-say about the paltry support their work receives. But researchers in these countries seldom reflect on whether they are doing their part to earn the money they so zealously demand.

In 2005, the annual budget of the Consejo Nacional de Ciencia y Tecnología, Mexico's main science-funding body, was about $\$ 800$ million. This amount pales in comparison to the budget of the US National Institutes of Health, which is 35 times larger at \$28 billion. But factor in the number of scientists in each country, and this difference in funding is not as substantial.

Depending on the source (the government or the scientists) and on the definition of scientist (academics doing any type of research or scientists receiving federal funds), there are quite disparate figures-between 10 and 100 times fewer scientists in Mexico than in the US. Thus, the amount of money available per scientist in the two countries turns out to be rather similar. In fact, a visit to almost any of the research institutes of the Universidad Nacional Autónoma de México, the largest university in the country and one of the largest in the world, illustrates this conclusion: the quality of their equipment rivals what one finds in US universities and is often better than what can be found in Europe.

Why is there such a large difference in the volume and impact of scientific output per capita between Mexico and the US? Mexican scientists are quick to point out many reasons besides the lack of money: the absence of a critical mass of researchers to create a strong scientific culture, the bureaucratic obstacles to importing reagents from abroad, the perceived discrimination of their work by scientific journals, and so on. Although these issues may be relevant to determining research output, there are other factors that must be taken into account to understand the scientific performance of countries like Mexico and the lack of governmental support for research.

First, in the US, postdocs drive the productivity of most labs. In Mexico, graduate and even undergraduate students bear the weight of most projects, as the country does not offer an academic atmosphere suitable to attract postdocs. Thus, differences in human, not financial, resources account for an important part of the gap between scientifically developed and emerging countries.
Some might argue that the difference in human resources simply mirrors the lack of available money-if there are no funds, how can we recruit postdocs? However, many US labs do not spend too much on postdocs, who often bring their own fellowships. Mexico itself has invested millions of dollars in postdoctoral fellowships of trainees who rarely stay in the country. Scientists should therefore switch their focus from blaming a lack of money for human resource development to creating an atmosphere that attracts both domestic and foreign postdocs.

Second, looking at the published scientific output of Mexico, it can be seen that many of the projects that have been funded lack originality. In many cases, they do not try to answer an original question but instead aim to provide evidence that an observation made by others is also true in one's favorite experimental system-'me-too' science.

This attitude, which has put a clamp on creative thinking and greatly limited the proliferation of innovative ideas, has not been seriously criticized by Mexican funding agencies or by the scientists themselves - no one ever wonders if what scientists are doing is novel enough to lead to a patent or to some clinical application. Ironically, this lack of interest has been partly fostered by the scientists themselves: any suggestion that they pay more attention to the applicability of what they do has always been received with hostility. The phrase 'applied science' is anathema for many Mexican scientists, and as a result, innovation has taken a back seat to the comfort of doing confirmatory science.

But times are changing, and the increased awareness of the commercial potential of biology is leading to a global push for translating academic research into technological advances. For institutions in the US, the potential of scientific discoveries to generate revenue has a priority higher than ever. In Mexico, technology-transfer offices and partnerships between academia and industry are virtually nonexistent. If scientists ask their government to invest more in science, is the government not entitled to ask what the return on its investment is?

This does not mean that basic science has no room in the modern world-quite the opposite, as it is basic research that often fuels technological advances. Nor does it mean that scientists in Mexico (and in other emerging nations that might recognize themselves in the above paragraphs) should accept the status quo and welcome whatever crumbs fall from the government's table. Researchers must continue to make their voices heard, but the verbal attacks and name-calling they sometimes use to make their case simply will not do. This strategy needs to give way to a new discourse on innovation and the benefits that come with it. This is a language that even 'a rancher' will understand. 